\title{
Nefrosis lipoidea en la infancia. Evolución y correlación anatomoclínica
}

\author{
M.C. Eugenio Rodríguez S. ${ }^{1}$; M.C. Alberto Vitlarroel S. ${ }^{1}$; M.C. Angela Delucchi B. ${ }^{1}$; \\ M.C. Franciseo Cano Sch. ${ }^{\prime}$; M.C. Helmar Rosenberg G. ${ }^{2}$; M.C. Federico Puga $C$.
}

\section{Lipoid nephrosis}

\begin{abstract}
Ljpoid nephrosis was identified by light, immunofluorescence and electron microscopy in 34 children aged 1 month to 10 years (23 males). These patients were followed up for a mean of 6.8 years (range 2.2 to 14 years). Treatment included oral prednisone ( $2 \mathrm{mg} \cdot \mathrm{kg} \cdot$ day for 4 weeks and then four days a week for 2 months). In steroid dependent of resistant patients oral cyclophosphamide $2.5 \mathrm{mg} \cdot \mathrm{kg}$ - day was given for 2 months. Satisfactory responses to steroid therapy were recorded in $73.5 \%$, steroid dependence in $23.5 \%$ and steroid resistance in $3 \%$ of cases respectively. As a whole, $97 \%$ of patients responded before 8 week to steroids, cyclosphosphamide or both. No morphologic differences were recorded from kidney biopsies among corticoid responders, dependents or resistants, neither between first and second biopsies which wese done in four steroid dependent and one steroid resistant children. Slight morphologic differences were observed depending on the time elapsed from initial symptoms to renal biopsy: 39 days $(\bar{x})$ when total disappearence of foot processes was seen (5 cases); 1 l month $(\bar{x})$ in 14 cases with partial pedicelar absence and 20 months $(\bar{x})$ in 15 chidren with segmental pedicelar absence altemating with zones of normal foot process morphology. The total number of nephrotic episodes was 149 , with a thean of 4.3 recurrences per patient, including three children (all girls) whose disease never recurred. Serious infections were detected in $4.7 \%$ of recurring episodes. At 5 years follow up $94 \%$ of patients were in cemission. No deaths occurred among these patients.
\end{abstract}

(Key words: Jipoid nephrosis, clinical course, prognosis.)

La nefrosis lipoidea (NL) es una glomerulopatia primaria cuya caracter istica principal a la microscopia electrónica es la desaparición de los pedicelos de las células epiteliales, sin alteración de la membrana basal y del mesangio, con normalidad estructural en la microscopja de luz e imunofluorescencia habitualmente negativa' ${ }^{\prime}$. Representa alrededor de $80 \%$ de los sindromes nefóticos que ocurren en niffos, evoluciona con recaídas frecuentes y periodos de remisión de meses a años. Excepcionalmente la enfermedad se manifesta por un solo episodio nefrótico.

El objetivo del presente trabajo fue determinar el tiempo en que evoluciona actualmente (con tratamiento) la enfermedad, comparado con la historia natural previa, no tratada. descrita por otras fuentes y conocer los cambios histopatológicos que ocurren en los diferentes momentos de su evolución, buscando un indice pronóstico.

1. Unidad de Nefrología. Hospital Luis Calvo Mackenna.

2 Departamento de Anatomía Patológica. Pontificia Universidad Católica de Chile.

\section{PACIENTES Y METODO}

Se incluye la información sobre 34 pacientes con NL que consultaron en la unidad de nefrología del hospital 1. Calvo Mackenna entre los aftos 1967 y 1987 . E] díagnóstico histológico se realizó mediante biopsia renal, con microscopía electrónica, de luz $\mathrm{e}$ inmunofluorescencia. in todos los casos se registraton edad, sexo, número de recaidas, repuesta a esteroides, inmunosupresores, o ambos, complicaciones, características morfológicas en la biopsia renal y duración de la enfermedad. Los pacientes fueron tratados con prednisona oral, $2 \mathrm{mg} \cdot \mathrm{kg} \cdot \mathrm{d}$ ía durante un mes $y$ con la misma dosis unitaria 4 veces por semana (de lines a jueves) durante 2 meses. En los pacientes córtico dependientes se empleó ciclofosfamida $2.5 \mathrm{mg} \cdot \mathrm{kg} \cdot \mathrm{d}$ ja en una dosis diaria durante 2 meses con recuento semanal de glóbulos blancos en sangre.

La NL se consideró sensible, dependiente a resistente a los esteroides según su respuesta clínica y de laborato. rio, de acuerdo a los criterios del "Estudio internacional de enfermedades del riñón en niños" (I.K.D.C.) ${ }^{3}$.

\section{RESULTADOS}

Treinta y cuatro pacientes con NL fueron seguidos por 2,2 a 14 años ( $x: 6,8$ años). De ellos, veintitrés eran varones $(67,6 \%)$. La relación hombre : mujer fue $2: 1$ y $91 \%$ tenían entre 1 y 6 
años de edad. Los síntomas se iniciaron entre las edades de 13 meses y 10 años.

Se registraron en total 115 reca idas: 0 a 2 ocurrieron en $35 \%$ de los niños afectados; 3 a 5 en $47 \%$ y 6 a 8 en $18 \%$. Tres pacientes presentaron un solo episodio de la enfermedad, todos eran mujeres. El total de episodios nefróticos fue 149 con un promedio de 4,3 recaídas por paciente. Ocho niffos presentaron cifras de presión arterial sobre el percentil 90 para la edad, en 7 esto ocurrió en el primer episodio, normalizándose en todos antes de las $\mathbf{4 8}$ horas, sin drogas antihipertensivas. Ningún paciente tuvo hematuria macroscópica durante la actividad o remisión de la enfermedad.

Fueron sensibles a los esteroides (prednisona) $73,5 \%$ de los casos, dependientes $23,5 \%$ y resistentes $3 \%$. La respuesta a los esteroides, a los inmunosupresores, a ambos, ocurrió antes de transcurrir ocho semanas en $97 \%$ de los pacientes (figura 1) y $94 \%$ se encontraban en remisión a los 5 años de iniciada la enfermedad (figura 2). En 4,7\% de los episodios se registraron complicaciones infecciosas, siempre durante la actividad de la enfermedad y ellas fueron: tres casos de bronconeumonia, dos de peritonitis, uno de celulitis y uno de erisipela. No hubo letalidad en esta serie.

En cinco pacientes, cuatro córtico-dependientes y uno córtico-resistente, se practicaron dos estudios histopatológicos a cada uno en diferentes tiempos de la enfermedad, encontrándose en ambas oportunidades las características morfológicas propjas de la NL (tabla 1).

\section{COMENTARIO}

La NL en pediatría continúa siendo la primera causa de síndrome nefrótico primario, y es asi que

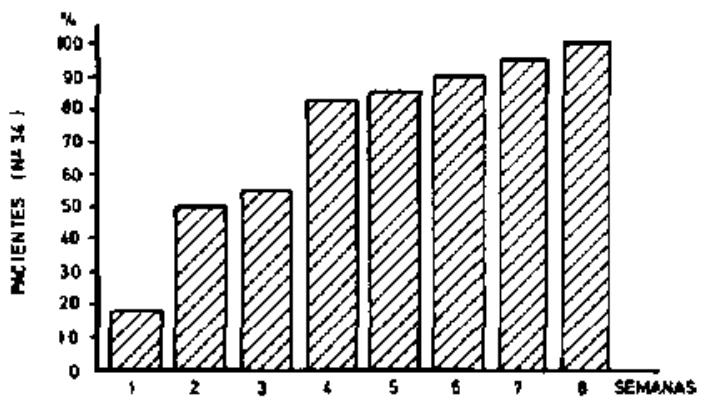

Figura 1:

Nefrosis lipoidea.

Tiempo de respuesta a tratamiento.

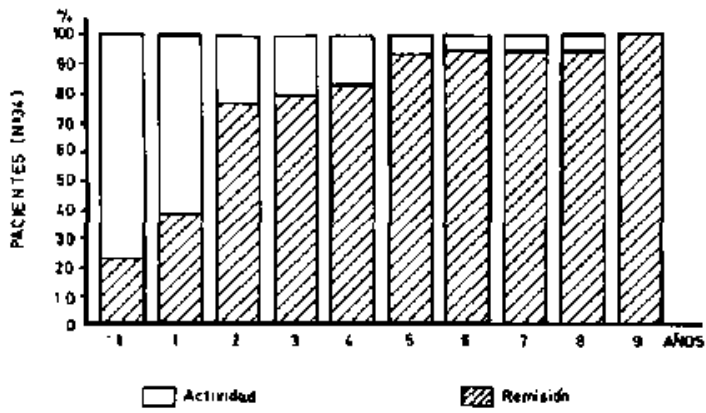

Figura 2:

Nefrosis lipoidea.

Duración de enfermedad activa.

en los últimos 10 años el diagnóstico en el nifio se realiza en base a las manifestaciones clínicas y de laboratorios, además de la respuesta al tratamiento esteroidal, lo que ha disminuido notablemente el número de biopsias renales 5 .

No existen diferencias entre la edad de presentación, el sexo, la historia natura] de la enfermedad $y$ la respuesta al tratamiento esteroidal $e$ inmunosupresor utilizado en esta serje con lo des: crito en la literatura ${ }^{2,5}$.

Tabla

Caracter ísticas morfológicas y tiempo de evolución de la nefrosis lipoidea

\begin{tabular}{ccl}
\hline n de casos & $\begin{array}{c}\text { Tiempo promedio de } \\
\text { enfermedad antes BR }\end{array}$ & \multicolumn{1}{c}{ Característica morfológica } \\
\hline 5 & 39 días & desaparición pedicelar completa \\
14 & 11 meses & $\begin{array}{l}\text { desaparición pedicelar parcelar } \\
\text { desaparición pedicelar segmentaria con zonas de } \\
\text { pedicelos normeles. }\end{array}$ \\
\hline
\end{tabular}

$\mathrm{BR}=$ Biopsia renal. 
El síndrome nefrótico con cambios histológicos mínimos en el nifo ha despertado gran interés entre los investigadores, legándose a la conclusión de que es benigno y tiene buen pronóstico. A pesar de permanecer activo durante muchos afos, no se asocia con deterioro de la función renal en la mayor parte de los casos descri$\operatorname{tos}^{2,3,6,7}$.

Se ha postulado que en la evolución morfo. lógica de la NL y otras glomerulopat ías primarias de pronóstico reservado podría suceder la transformación de una a otra forma, opinión sobre la cual hay aún mucha controversia ${ }^{9,12}$.

La enfermedad cursa con recaídas, siendo lo más frecuente que ocurran más de 3 y menos de 5 , pero excepcionalmente está constituida por un solo episodio. Ocasionalmente puede aparecer hipertensión arterial al injicio, como en algunos de los casos de esta serie, y también microhematuria $^{4,5}$. No se ha descrito hematuria macroscópica.

La resistencia a los corticoides se observó sólo en 3\% de los casos, planteándose en ellos el uso de inmunosupresores, criterio que ha cambiado si se compara con casuisticas anteriores, pues actualmente éstos se proponen después de la tercera re. caída, y en los pacientes córtico-dependientes su empleo no constituye ahora indicación de biopsia renal ${ }^{4,5,7}$.

Las recaídas frecuentes o la córtico-dependencia no implican đaño renal o evolución hacia la insuficiencia renal. No se apreciaron diferencias histopatológicas en los pacientes sensibles, dependientes o resistentes. En aquellos con biopsias repetidas se observaron sólo diferencias morfológicas sutiles, dependientes del tiempo de evolución de la enfermedad.

Esta serie describe la duración de la $\mathrm{NL}$ tratada, demostrando que en más de $90 \%$ es inferior a 5 años, lo que coincide con la evolu. ción natural de la enfermedad no tratada.

La NL tiene excepcionalmente complicacio. nes, siendo las más frecuentes las infecciosas, que aparecen durante los episodios nefróticos y se atribuyen a trastornos de la inmunidad ${ }^{13,14}$.

La evolución natural de la enfermedad en el niño no ha cambiado. El uso de esteroides e inmunosupresores ha logrado acortar la duración de los episodios y los antibióticos han permitido tratar las complicaciones, con lo cual ha mejorado el pronóstico de la enfermedad. Estos resultados reafirman la idea de que su evolución es benigna, pues no conduce a la insuficiencia renal y su tratamiento no produce complicaciones $\mathrm{nj}$ secuelas importantes.

\section{RESUMEN}

Se presentan 34 pacientes; 23 hombres, con diagnóstico de nefrosis lipoidea (NL) confirmado con biopsia renal. El promedio de seguimiento fue 6,8 años (rango 2,2 a 14 años); $97 \%$ de pacientes respondieron satisfactoriamente a los esteroides, a los inmunosupresores o ambos. Durante la actividad de la enfermedad $4,7 \%$ de los episodios se complicaron con infecciones graves; $94 \%$ de los pacientes estaban en remisión a los 5 anos de iniciada la enfermedad. Las lesiones morfológicas renales varían con el tiempo de evolución ( $\overline{\mathrm{x}} 39$ días en 5 casos con desaparición completa de los pedicelos, 11 meses en 14 casos con desaparición parcelar y 20 meses en 15 casos con zonas de desaparición segmentaria alternando con otras con pedicelos normales). No se observaron diferencias significativas entre 2 biopsias en cinco pacientes dependientes $(n=4) 0$ resis. tentes $(n=1)$ a los corticosteroides a quienes se practicaron biopsias renales. No hubo mortalidad en esta serie ni deterioro de la función renal.

(Palabras claye: nefrosis lipoidea, nefrosis con lesiones mínimas, síndrome nefrótico.)

\section{REFERENCIAS}

1. Rosenberg. H.: Primary glomerular diseases (Primary glomerulopathies), Pat Res Pract 1986; 181: 489-523.

2. Koskimies, O.; Vilska. J.; Ropola, J,; Hallman, $N$.: Long-term autcome of primary nephrotic syndrome. Arch Dis Child 1982; $57: 544-548$.

3. Intenational study of kidney Disease in Children: The primary nephrotic syndrome in children. Identification of patiens with minimal change nephrotic syndrome from initial response to prednisone. J Pediatr 1981 ; 98: 561-564.

4. Schnoper, H,W, Robson, A.M.: Nephrotic syndrome: Minimal change disease, focal glomerulosclerosis, and related disorders. En: Schrier, R.W.; Gottschalk, C. ed. Diseases of the kidney. Part. II Boston: Little, Brown and Company 1988; 1949 1990.

5. Puga, F,; Rodriguez, $E$.; Rosenberg, $H$.: Síndrome nefrótico idiogático III parte: Tratamiento y pronóstico. Rev Chil Pediate 1974; $45: 217-220$.

6. Trompeter, R.S.; Hicks, J.; Lloyd, B.W.; White, R.H.R.; Cameron, J.S.: Long-term outcome for children with minimal change nephrotic syndrome. Lancet 1985; 1 : 368-370. 
7. Cameron, $J$.: The long-term outcome of glomerular diseases. Fn: Schrier, R.W,; Gottschalk, C. ed. Diseases of the kidney. Part II. Boston: Little, Brown and Company 1988; 2127-2180.

8. Border, $W$.: Distinguishing minimal - change disease from mesangial disorders. Kidney Int 1988: 34 : $419-434$.

9. Tejari, A.: Morphological transition in minimal change nephrotic syndrome. Nephron 1985; 39: $157-159$.

10. Silva F.: Overview of pediatric nephropathology. Kidney int. $1988: 33: 1016-1032$.

11. International Study of Kidney Disease in Chilaren: Minimal change nephrotic syndrome in children:
Deaths during the first 5 to 15 years observation. Pediattics 1984; 73: 497-501.

12. Kashtan $C_{1}:$ Melvin, $T$; Kim, Y:;Long-term followup of patients with steroid-dependent, minimal change nephrotic syndrome. Clin Nephrol 1988; 29: 79-85,

13. Bernard, D.: Extrarenal complications of the nephrotic syndrome. Kidney Int 1988; 33: 1184 . 1202.

14. Fodor, P.; Saitia, M.; Rodríguez, E.: Gonzälez, B.; Schlesinger. $I_{.}: \mathrm{T}$ cell dysfunction in minimal change nephrotic syndrome of chilhood. Am J Dis Child 1982; 136: 713-717. 